\title{
POWERS OF POSITIVE POLYNOMIALS AND CODINGS OF MARKOV CHAINS ONTO BERNOULLI SHIFTS
}

\author{
BRIAN MARCUS AND SELIM TUNCEL
}

(Communicated by Klaus Schmidt)

\begin{abstract}
We give necessary and sufficient conditions for a Markov chain to factor onto a Bernoulli shift (i) as an eventual right-closing factor, (ii) by a right-closing factor map, (iii) by a one-to-one a.e. right-closing factor map, and (iv) by a regular isomorphism. We pass to the setting of polynomials in several variables to represent the Bernoulli shift by a nonnegative polynomial $p$ in several variables and the Markov chain by a matrix $A$ of such polynomials. The necessary and sufficient conditions for each of (i)-(iv) involve only an eigenvector $r$ of $A$ and basic invariants obtained from weights of periodic orbits. The characterizations of (ii)-(iv) are deduced from (i). We formulate (i) as a combinatorial problem, reducing it to certain state-splittings (partitions) of paths of length $n$. In terms of positive polynomial masses associated with paths, the aim then becomes the construction of partitions so that the masses of the paths in each partition element sum to a multiple of $p^{n}$, the multiple being prescribed by $r$. The construction, which we sketch, relies on a description of the terms of $p^{n}$ and on estimates of the relative sizes of the coefficients of $p^{n}$.
\end{abstract}

\section{INTRODUCTION}

We will describe in this article several recent results for coding Markov chains onto Bernoulli shifts. Adopting an approach first espoused by Parry and Tuncel, we will associate polynomial matrices to Markov chains and translate the jointly measure-theoretic and topological coding problems into combinatorial ones. Bernoulli shifts will then be represented by polynomials $p$ with nonnegative integral coefficients, and we will find ourselves facing questions about powers of $p$ : We start our exposition with a number of results on the structure and coefficients of large powers of positive polynomials in several variables. This material is isolated in section 2, and readers whose interests lie with powers of polynomials may go directly to section 2. We remark that proofs of the results in section 2 rely, in turn, on ideas from ergodic theory, specifically, the notion of entropy at a weight-per-symbol and the related equilibrium states [MT2].

It is well known from Ornstein's isomorphism theory [O] that entropy is a complete invariant of measure-theoretic isomorphism for mixing Markov chains. In that result the isomorphism is two-sided, meaning that it and its inverse are allowed to use the entire past and the entire future of a point in order to determine the present coordinate of its image. In a one-sided isomorphism, the isomorphism

Received by the editors January 21, 1999.

1991 Mathematics Subject Classification. Primary 28D20; Secondary 11C08, 05A10.

Partially supported by NSF Grant DMS-9622866. 
and its inverse are allowed to use the entire past, but none of the future. In [AMT] Markov chains were completely classified up to one-sided isomorphism, via an effective algorithmic procedure. An intermediate notion is regular isomorphism. Here, the isomorphism and its inverse are allowed to use the entire past and a uniformly bounded amount of the future.

Ideas emanating from symbolic dynamics, in particular right-resolving and rightclosing maps of shifts of finite type (see below for definitions), have a strong bearing on regular isomorphism and one-sided isomorphism of Markov chains: If a rightclosing map is one-to-one almost everywhere, then it defines a (rather concrete) regular isomorphism between the shifts with respect to their measures of maximal entropy. More generally, if the shifts of finite type are endowed with Markov measures and the map preserves measure, then we obtain a regular isomorphism between the Markov chains. It was shown in [BT] that two Markov chains are regularly isomorphic if and only if there exists a Markov chain which factors onto each of them by a right-closing map which is one-to-one almost everywhere. Thus, the study of regular isomorphism largely reduces to that of right-closing maps between Markov chains.

Likewise, two Markov chains are one-sidedly isomorphic if and only if there exists a Markov chain which factors onto each of them by a right-resolving map which is one-to-one almost everywhere. This fact serves as the first step of the classification of [AMT]. Right-closing maps form the closure of right-resolving ones under block isomorphism, that is, measure-preserving topological conjugacy.

We will give complete answers to the following questions for an arbitrary Markov chain and a Bernoulli shift.

(1) Does the Markov chain eventually factor onto the Bernoulli shift by rightclosing maps?

(2) Does the Markov chain factor onto the Bernoulli shift by a right-closing map? By a right-closing map which is one-to-one almost everywhere?

(3) Is the Markov chain regularly isomorphic to the Bernoulli shift?

Without consideration of measures or, equivalently, restricting attention to measures of maximal entropy, analogues of (1)-(3) were answered for shifts of finite type in the sequence of papers [M, BMT, A]. It is natural to seek generalizations of these results to Markov chains. The work presented here realizes the generalizations for a Bernoulli image.

Definitions and the relationship between Markov chains and matrices of polynomials are recalled in section 3 below. The answers to (1)-(3) are then presented in section 4 . Sections 5 and 6 outline their proofs. Section 5 reduces (1)-(3) to (1) and, for (1), formulates the problem in terms of state-splittings with certain combinatorial properties. Section 6 is concerned with the construction of the desired state-splitting. Details and complete proofs may be found in [MT4] and [MT5].

\section{Powers of Positive polynomials}

Let $p$ be a Laurent polynomial in the variables $x_{1}, \ldots, x_{k}$. For $w=\left(w_{1}, \ldots, w_{k}\right) \in$ $\mathbb{Z}^{k}$, write $x^{w}=x_{1}^{w_{1}} \cdots x_{k}^{w_{k}}$ and denote the coefficient of $x^{w}$ in $p$ by $p_{w}$. Then $p=\sum_{w \in \mathbb{Z}^{k}} p_{w} x^{w}$ and $p_{w}$ are nonzero for only finitely many $w \in \mathbb{Z}^{k}$. Let $\log (p)=$ $\left\{w \in \mathbb{Z}^{k}: p_{w} \neq 0\right\}$. The Newton polyhedron $W(p)$ of $p$ is given by the rational convex hull of $\log (p)$. Denote the collection of nontrivial faces of $W(p)$ by $\mathcal{F}(p)$. 
For $F \in \mathcal{F}(p)$, let

$$
p_{F}=\sum_{w \in F} p_{w} x^{w},
$$

and think of $p_{F}$ as the $F$-face of $p$. Let $\Delta(p)$ be the subgroup of $\mathbb{Z}^{k}$ generated by $\{a-b: a, b \in \log (p)\}$, and pick any $c_{p} \in \log (p)$ to distinguish a coset $c_{p}+\Delta(p)$ of $\Delta(p)$.

It is easy to see that $\log \left(p^{n}\right) \subset n W(p) \cap\left(n c_{p}+\Delta(p)\right)$. If $H \geq 0$ and $S \subset \mathbb{R}^{k}$, we use the usual norm and distance in $\mathbb{R}^{k}$ to write $B(S, H)=\left\{w \in \mathbb{R}^{k}: \operatorname{dist}(w, S)<\right.$ $H$ \}. For $F \in \mathcal{F}(p)$ we denote by $\partial F$ the boundary of $F$ taken in its affine hull. Equivalently, $\partial F$ is the union of all $G \in \mathcal{F}(p)$ which are proper subsets of $F$. We put

$$
\operatorname{Int}\left(p^{n}, H\right)=\left(W\left(p^{n}\right) \backslash B\left(\partial W\left(p^{n}\right), H\right)\right) \cap\left(n c_{p}+\Delta(p)\right) .
$$

In other words, $\operatorname{Int}\left(p^{n}, H\right)$ is the set of elements of $n c_{p}+\Delta(p)$ remaining in $W\left(p^{n}\right)=$ $n W(p)$ after we remove a band of width $H$ from around the boundary. For $p \in$ $\mathbb{R}^{+}\left[x_{1}^{ \pm}, \ldots, x_{k}^{ \pm}\right]$, we find that there exists $H(p) \geq 0$ such that $\operatorname{Int}\left(p^{n}, H(p)\right) \subset$ $\log \left(p^{n}\right)$ for all $n \in \mathbb{N}$. The following then describes $\log \left(p^{n}\right)$ for large $n$.

Theorem 2.1. Let $p \in \mathbb{R}^{+}\left[x_{1}^{ \pm}, \ldots, x_{k}^{ \pm}\right]$. Suppose that, for each $F \in \mathcal{F}(p)$, we have constants $K_{F} \geq H\left(p_{F}\right)$ and $D_{F} \geq 0$. Then there exist $H_{F} \geq K_{F}, N \in \mathbb{N}$ and finite subsets $T(F) \subset \mathbb{Z}^{k}$ such that $T(W(p))=\{0\}$ and

$$
\log \left(p^{n}\right)=\bigcup_{F \in \mathcal{F}(p)}\left(\operatorname{Int}\left(p_{F}^{n}, H_{F}+D_{F}\right)+T(F)\right)=\bigcup_{F \in \mathcal{F}(p)}\left(\operatorname{Int}\left(p_{F}^{n}, H_{F}\right)+T(F)\right)
$$

for all $n \geq N$.

In particular, other than magnification of the materials used, the sets $\log \left(p^{n}\right)$ are built in the same way: For each $F \in \mathcal{F}(p)$ cut from the lattice $\Delta\left(p_{F}\right)$ (or its copy $\left.n c_{p_{F}}+\Delta\left(p_{F}\right)\right)$ exactly $|T(F)|$ pieces of the right size in the shape of $W\left(p_{F}\right)$, translate them according to $T(F)$, and glue them together.

The proof of (2.1) is comprised of two stages. The first expresses $\log \left(p^{n}\right)$ in terms of a union over facets of $W(p)$. The second refines this by applying an induction based on the (codimension of) the faces of $W(p)$.

Now write $R_{k}=\mathbb{Z}\left[x_{1}^{ \pm}, \ldots, x_{k}^{ \pm}\right]$and $R_{k}^{+}=\mathbb{Z}^{+}\left[x_{1}^{ \pm}, \ldots, x_{k}^{ \pm}\right]$. Let $p \in R_{k}^{+}$and, for $F \in \mathcal{F}(p)$, put $c_{F}=c_{p_{F}}$. In sections 5 and 6 we will need the following three estimates on the relative sizes of coefficients of $p^{n}$ for large $n$.

Proposition 2.2. Let $p \in \mathbb{Z}^{+}\left[x_{1}^{ \pm}, \ldots, x_{k}^{ \pm}\right]$and let $F$ be a proper face of $W(p)$. Let $L \in \mathbb{N}$ and $K, \bar{\epsilon}_{F}>0$. There exist $\epsilon_{F}>0$ and $N \in \mathbb{N}$ such that for $a \in \mathbb{Z}^{k}$ the inequality

$$
\left(p^{n}\right)_{a+b} \geq K\left(p^{n}\right)_{a}
$$

holds whenever $n \geq N, \frac{a}{n} \in B\left(F, \epsilon_{F}\right) \backslash B\left(\partial F, \bar{\epsilon}_{F}\right)$ and $b \in\left(\log \left(p^{L}\right) \backslash \log \left(p_{F}^{L}\right)\right)-$ $\log \left(p_{F}^{L}\right)$.

Proposition 2.3. Let $p \in \mathbb{Z}^{+}\left[x_{1}^{ \pm}, \ldots, x_{k}^{ \pm}\right]$and $F \in \mathcal{F}(p)$. Let $\bar{\epsilon}_{F}, \theta>0$ and $D \in \mathbb{N}$. There exist $\epsilon_{F}>0$ and $N \in \mathbb{N}$ such that

$$
\left|\frac{\left(p^{n}\right)_{a} /\left(p^{n}\right)_{a+b}}{\left(p^{n}\right)_{a+u} /\left(p^{n}\right)_{a+u+b}}-1\right|<\theta
$$


whenever $n \geq N$ and $a, b, u \in \mathbb{Z}^{k}$ are such that $a, a+u \in \log \left(p^{n}\right), b \in \Delta\left(p_{F}\right)$, $\frac{a}{n} \in B\left(F, \epsilon_{F}\right) \backslash B\left(\partial F, \bar{\epsilon}_{F}\right)$, and $\|b\|,\|u\| \leq D$.

Proposition 2.4. Let $p \in \mathbb{Z}^{+}\left[x^{ \pm}, \ldots, x_{k}^{ \pm}\right]$and $F \in \mathcal{F}(p)$. Let $\epsilon_{F}>0, l \in \mathbb{Z}$ and $D \in \mathbb{N}$. There exist $\epsilon_{F}>0$ and $K, N \in \mathbb{N}$ such that

$$
\frac{1}{K}\left(p^{n+l}\right)_{a+l c_{F}+b} \leq\left(p^{n}\right)_{a} \leq K\left(p^{n+l}\right)_{a+l c_{F}+b}
$$

whenever $n \geq N$ and $a, b \in \mathbb{Z}^{k}$ are such that $\frac{a}{n} \in B\left(F, \epsilon_{F}\right) \backslash B\left(\partial F, \bar{\epsilon}_{F}\right), b \in \Delta\left(p_{F}\right)$ and $\|b\| \leq D$.

Details of (2.1)-(2.4) may be found in [MT4]. In the case of (2.2)-(2.4), the method of proof and the more technical results at its basis may be objects of greater general interest. They involve the notion of entropy at a weight-per-symbol and the corresponding equilibrium states, which are imported from [MT2] and strengthened in the Bernoulli setting: Letting $W_{\mathbb{R}}(p)$ denote the real convex hull of $\log (p)$ and $d=\sum_{w \in \mathbb{Z}^{k}} p_{w}$, it turns out that the map $w \mapsto s(w)$ that sends $w \in W_{\mathbb{R}}(p)$ to the corresponding equilibrium distribution $s(w)$, which is a probability vector of size $d$, is $C^{1}$ on $W_{\mathbb{R}}(p)$. It is also shown that, for small $\epsilon_{F}, \delta>0$ and large $n$, multinomial coefficients lying in a $\delta$-ball around $w=\frac{a}{n}$ will estimate $\left(p^{n}\right)_{a}$ uniformly closely for $w \in B\left(F, \epsilon_{F}\right) \backslash B\left(\partial F, \bar{\epsilon}_{F}\right)$. From this common ground, the proof of each of (2.2)(2.4) is reduced to a calculation involving multinomial coefficients.

As far as we are aware, the $F$-faces $p_{F}$ were introduced by Handelman $[\mathrm{H}]$.

\section{MARKOV CHAINS AND POLYNOMIAL MATRICES}

It is well known that a directed graph $G$ defines a shift of finite type $\left(X_{G}, \sigma_{G}\right)$. A shift-commuting continuous surjection $\phi: X_{G} \rightarrow X_{H}$ of shifts of finite type is known as a factor map. The map $\phi$ is right-closing if left-asymptotic distinct points of $X_{G}$ have distinct images under $\phi$. Right-closing maps are bounded-to-one and, assuming $G$ is irreducible, there exists $d=d(\phi) \in \mathbb{N}$, called the degree of $\phi$, so that every doubly transitive $y \in X_{H}$ has exactly $d$ pre-images. If $d=1$, the map $\phi$ is said to be one-to-one almost everywhere. For these and other basic definitions and facts on shifts of finite type and factor maps, see [LM].

The directed graph $G$ is weighted if, for some multiplicative Abelian group $\mathcal{G}$, each edge $e$ of $G$ is assigned a weight $\operatorname{wt}_{G}(e) \in \mathcal{G}$. The weight of a path $\gamma=$ $e_{1} e_{2} \cdots e_{n}$ is the product $\mathrm{wt}_{G}(\gamma)=\mathrm{wt}_{G}\left(e_{1}\right) \cdots \mathrm{wt}_{G}\left(e_{n}\right)$. Let $\Gamma(G)$ be the subgroup of $\mathcal{G}$ generated by weights of cycles, and let $\Delta(G)$ be the subgroup of $\Gamma(G)$ consisting of ratios $\mathrm{wt}_{G}(\gamma) / \mathrm{wt}_{G}(\eta)$ of weights of cycles $\gamma, \eta$ of the same length. For irreducible $G$ of period $d$, let $\gamma, \eta$ be cycles whose lengths satisfy $l(\gamma)=l(\eta)+d$ and use $c_{G}=\mathrm{wt}_{G}(\gamma) / \mathrm{wt}_{G}(\eta)$ to distinguish a coset $c_{G} \Delta(G)$. (For properties of $\Gamma, \Delta, c \Delta$ see [PS, MT1].)

The graphs we consider will always be directed, irreducible and, except for a brief spell, weighted by monomials. Such graphs correspond to irreducible matrices $A$ over $R_{k}^{+}$: Express each entry $A(I, J)$ as a sum (with multiplicity) of monomials, place as many edges from $I$ to $J$ as there are terms in this sum, and bijectively assign the monomials as weights. Confusing $A$ with the associated graph $G(A)$, we write $\mathrm{wt}_{A}(\gamma), \Gamma(A), \Delta(A), c_{A} \Delta(A)$ for the objects defined above for $G(A)$. For a $G(A)$-cycle $\gamma$ of length $l=l(\gamma)$, we define the weight-per-symbol of $\gamma$ to be $\operatorname{wps}_{A}(\gamma)=\frac{1}{l} \log \left(\operatorname{wt}_{A}(\gamma)\right) \in \mathbb{Q}^{k}$. The rational convex hull of $\left\{\operatorname{wps}_{A}(\gamma): \gamma\right.$ cycle of $G(A)\}$ is the weight-per-symbol polytope $W(A)$ of $A$. For $x_{1}, \ldots, x_{k}>0$, let 
$\beta_{A}\left(x_{1}, \ldots, x_{k}\right)>0$ be the spectral radius of the nonnegative real-valued matrix $A\left(x_{1}, \ldots, x_{k}\right)$. Writing $\mathbb{R}^{++}$for the positive reals, this defines the beta function $\beta_{A}:\left(\mathbb{R}^{++}\right)^{k} \rightarrow \mathbb{R}^{++}$of $A$.

We write $\left(X_{A}, \sigma_{A}\right)$ for the shift of finite type defined by $G(A)$ together with the weights-per-symbol assigned to its periodic orbits. We require finite-to-one factor maps $\phi: X_{A} \rightarrow X_{B}$ between such systems to be weight-preserving in that $\operatorname{wps}_{B}(\phi(\gamma))=\operatorname{wps}_{A}(\gamma)$ for every periodic orbit $\gamma$ of $\left(X_{A}, \sigma_{A}\right)$. The (clearly necessary) factor periodic point condition for $A, B$ is the requirement that, for each periodic orbit $\gamma$ of $\left(X_{A}, \sigma_{A}\right)$, there exist in $\left(X_{B}, \sigma_{B}\right)$ a periodic orbit of the same weight-per-symbol whose period divides that of $\gamma$.

Applying the above to $p \in R_{k}^{+}$by viewing it as a $1 \times 1$ matrix, note that there is a slight clash with the definitions of $\Gamma(p), \Delta(p), c_{p} \Delta(p)$ given in section 2, resulting from confusing monomials and their exponents.

Markov chains are traditionally defined by stochastic matrices. Given an irreducible stochastic matrix $M$, we construct a graph $G(M)$ taking weights in $\mathbb{R}^{++}$: The vertices of $G(M)$ are given by the indexing set of $M$, and there is an edge of weight $M(I, J)$ from $I$ to $J$ whenever $M(I, J)>0$. The shift of finite type $\left(X_{M}, \sigma_{M}\right)$ defined by $G(M)$ and the Markov measure $\mu_{M}$ defined by $M$ give us the Markov chain $\left(X_{M}, \sigma_{M}, \mu_{M}\right)$. We will write $\mathrm{wt}_{M}(\gamma), \Gamma(M), \Delta(M), c_{M} \Delta(M)$ for the objects defined for the weighted graph $G(M)$. For $t \in \mathbb{R}$, let $\beta_{M}(t)$ be the spectral radius of the matrix $M^{(t)}$ with

$$
M^{(t)}(I, J)= \begin{cases}0 & \text { if } M(I, J)=0 \\ M(I, J)^{t} & \text { if } M(I, J)>0 .\end{cases}
$$

This defines the beta function $\beta_{M}: \mathbb{R} \rightarrow \mathbb{R}^{++}$of $M$.

Letting $Q$ also be an irreducible stochastic matrix, we require factor maps between $\left(X_{M}, \sigma_{M}, \mu_{M}\right)$ and $\left(X_{Q}, \sigma_{Q}, \mu_{Q}\right)$ to be measure-preserving. In particular, $\left(X_{Q}, \sigma_{Q}, \mu_{Q}\right)$ is a right-closing factor of $\left(X_{M}, \sigma_{M}, \mu_{M}\right)$ if there exists a right-closing map $\phi$ of the shift of finite type $\left(X_{M}, \sigma_{M}\right)$ onto $\left(X_{Q}, \sigma_{Q}\right)$ such that $\mu_{M} \circ \phi^{-1}=\mu_{Q}$. The Markov chain $\left(X_{Q}, \sigma_{Q}, \mu_{Q}\right)$ is a right-closing eventual factor of $\left(X_{M}, \sigma_{M}, \mu_{M}\right)$ if $\left(X_{Q}, \sigma_{Q}^{n}, \mu_{Q}\right)$ is a right-closing factor of $\left(X_{M}, \sigma_{M}^{n}, \mu_{M}\right)$ for all large $n$.

Suppose the rows of $Q$ are identical, so that $\left(X_{Q}, \sigma_{Q}, \mu_{Q}\right)$ is a Bernoulli shift. Also suppose $\beta_{M}=\beta_{Q}$, since this equality is necessary for the coding problems under consideration. Then, by $(6.1)$ of $[\mathrm{MT} 1], \Gamma(M) \subset \Gamma(Q)$. Pick a basis $a_{1}, \ldots, a_{k}$ of the finitely generated Abelian subgroup $\Gamma(Q)$ of $\mathbb{R}^{++}$. Upon conjugating $M, Q$ by suitable diagonal matrices, we obtain matrices whose entries lie in $\Gamma(Q)$. Hence, each nonzero entry of $M, Q$ can be uniquely expressed as a product of integral powers of $a_{1}, \ldots, a_{k}$. Replacing each $a_{i}$ by an indeterminate $x_{i}$, we get from $M, Q$ irreducible matrices $A, B$ over $R_{k}^{+}$. A finite-to-one factor map $\phi$ of the shift of finite type $\left(X_{M}, \sigma_{M}\right)$ onto $\left(X_{Q}, \sigma_{Q}\right)$ satisfies $\mu_{M} \circ \phi^{-1}=\mu_{Q}$ if and only if $\phi$ is a (weight-preserving) factor map of $\left(X_{A}, \sigma_{A}\right)$ onto $\left(X_{B}, \sigma_{B}\right)$. (See section 2 of $[\mathrm{T}]$ for details and credits.) Moreover, since the rows of $Q$ are identical, we can put $p=\sum_{J} B(I, J)$ for any row $I$ to identify $\left(X_{B}, \sigma_{B}\right)$ with $\left(X_{p}, \sigma_{p}\right)$.

Thus, we are in the position of having $p \in R_{k}^{+}$and an irreducible $R_{k}^{+}$-matrix $A$ with $\beta_{A}=p$ and studying weight-preserving maps of $\left(X_{A}, \sigma_{A}^{n}\right)$ onto $\left(X_{p}, \sigma_{p}^{n}\right)$. The equality $\beta_{A}=p$ implies that $W(A)=W(p)$, the Newton polyhedron of $p$. Consider $F \in \mathcal{F}(p)$. Define a subgraph of $G(A)$ by retaining an edge $e$ of $G(A)$ (and its weight $\mathrm{wt}_{A}(e)$ ) if and only if there exists a $G(A)$-cycle $\gamma$ which contains $e$ and has $\operatorname{wps}_{A}(\gamma) \in F$. The resulting subgraph is nonwandering; that is, it consists 
only of irreducible components, which we call the $F$-components of $A$. The beta function of an $F$-component either equals $p_{F}$ or it is strictly less than $p_{F}$ on all of $\left(\mathbb{R}^{++}\right)^{k}$; the $F$-components in the former category are said to be principal. (See [MT1, T] for the details.) $F$-components of $A$ will be central to our solutions of (1)-(3) of the introduction. Note that when $F=W(p)$, we have a single (principal) $W(p)$-component, namely, $A$ itself.

\section{The CODING RESUlts}

To formulate the conditions that will feature in the solutions of (1)-(3), let $p \in R_{k}^{+}$and let $F$ be a proper face of $W(p)$. A polynomial $q \in R_{k}$ will be said to $F$-conform to $p$ if there exists $n \in \mathbb{N}$ so that for some $u \in \mathbb{Z}^{k}$ we have

$$
\log (q)+u \subset \log \left(p^{n}\right) \text { and }(\log (q)+u) \cap \log \left(p_{F}^{n}\right) \neq \emptyset
$$

in this case we will put $\log _{F}(q)=\log (q) \cap\left(\log \left(p_{F}^{n}\right)-u\right)$ and $q_{F}=\sum_{w \in \log _{F}(q)} q_{w} x^{w}$. Roughly speaking, $F$-conformity asks that, at and near $F$, both the "shape" and the "gap structure" of $\log (q)$ be subordinate to $\log \left(p^{n}\right)$. For a group $\Delta$ of monomials in $R_{k}$, we put $R(\Delta)=\left\{q \in R_{k}: \log (q) \subset \log (\Delta)\right\}$.

Let $A$ be an irreducible $R_{k}^{+}$-matrix with $\beta_{A}=p$. By a right eigenvector of $A$ we will mean a nontrivial vector $r$ over $R_{k}$ such that $A r=p r$. Our results will require a right eigenvector which satisfies three conditions. The first two are:

(a) The entries of $r$ lie in $R_{k}^{+}$.

(b) For each proper face $F$ of $W(p)$ and each state $I$ that belongs to an $F$ component of $A$, the entry $r(I) F$-conforms to $p$.

The existence of a right eigenvector satisfying (a) was proved in [MT3]. For the description of the third condition, let $F \in \mathcal{F}(p)$, let $\mathcal{C}$ be a principal $F$-component of $A$, and let $A_{\mathcal{C}}$ be the matrix representing $\mathcal{C}$. Using (b), let $r_{\mathcal{C}}$ be the vector indexed by the states of $\mathcal{C}$ and having $r_{\mathcal{C}}(I)=r(I)_{F}$ for $I \in \mathcal{C}$. We then find a monomial $m$ and a diagonal matrix $D$ with monomials for its diagonal entries such that $\widetilde{p}_{F}=m p_{F} \in R\left(\Delta\left(p_{F}\right)\right), \widetilde{A}_{\mathcal{C}}=m D A_{\mathcal{C}} D^{-1}$ is over $R\left(\Delta\left(A_{\mathcal{C}}\right)\right) \subset R\left(\Delta\left(p_{F}\right)\right)$, $\widetilde{r}_{\mathcal{C}}=D r_{\mathcal{C}}$ is over $R\left(\Delta\left(p_{F}\right)\right)$ and $\widetilde{A}_{\mathcal{C}} \widetilde{r}_{\mathcal{C}}=\widetilde{p}_{F} \widetilde{r}_{\mathcal{C}}$. Put $R_{p_{F}}=R\left(\Delta\left(p_{F}\right)\right)\left[\frac{1}{\tilde{p}}\right]$, the ring obtained upon adjoining $\frac{1}{\widetilde{p}}$ to $R\left(\Delta\left(p_{F}\right)\right)$. Letting $d$ denote the period of $\mathcal{C}$, we have the subring $R_{A_{\mathcal{C}}}=R\left(\Delta\left(A_{\mathcal{C}}\right)\right)\left[\frac{1}{\widetilde{p}^{d}}\right]$ of $R_{p_{F}}$. View $R_{p_{F}}$ as an $R_{A_{\mathcal{C}}}$-module. For a period class $C$ of $\mathcal{C}$, let $\widetilde{r}_{C}$ be the restriction of $\widetilde{r}_{\mathcal{C}}$ to $C$ and consider the $R_{A_{\mathcal{C}}}$ submodule $\left\langle r_{C}\right\rangle$ of $R_{p_{F}}$ generated by the entries of $\widetilde{r}_{C}$. Our third requirement is that $\left\langle r_{C}\right\rangle$ equal $R_{p_{F}}$ :

(c) If $F \in \mathcal{F}(p)$ and $C$ is a period class of a principal $F$-component $\mathcal{C}$ of $A$, then the $R_{A_{\mathcal{C}}}$-module $\left\langle r_{C}\right\rangle$ equals $R_{p_{F}}$.

The following results answer (1)-(3) of the introduction.

Theorem 4.1. Let $\left(X_{M}, \sigma_{M}, \mu_{M}\right)$ be a Markov chain and $\left(X_{Q}, \sigma_{Q}, \mu_{Q}\right)$ a Bernoulli shift. Then $\left(X_{Q}, \sigma_{Q}, \mu_{Q}\right)$ is a right-closing eventual factor of $\left(X_{M}, \sigma_{M}, \mu_{M}\right)$ if and only if $\beta_{M}=\beta_{Q}$ and, considering the $R_{k}^{+}$-matrix $A$ and polynomial $p \in R_{k}^{+}$associated with $M, Q$, there exists a right eigenvector $r$ of $A$ satisfying (a)-(c).

Theorem 4.2. Let $\left(X_{M}, \sigma_{M}, \mu_{M}\right)$ be a Markov chain and $\left(X_{Q}, \sigma_{Q}, \mu_{Q}\right)$ a Bernoulli shift. Then $\left(X_{Q}, \sigma_{Q}, \mu_{Q}\right)$ is a right-closing factor of $\left(X_{M}, \sigma_{M}, \mu_{M}\right)$ if and only if $\beta_{M}=\beta_{Q}$, and, considering the $R_{k}^{+}$-matrix $A$ and polynomial $p \in R_{k}^{+}$associated 
with $M, Q$, there exists a right eigenvector $r$ of $A$ satisfying (a)-(c) and the factor periodic point condition holds for $A, p$. Moreover, $\left(X_{Q}, \sigma_{Q}, \mu_{Q}\right)$ is a factor of $\left(X_{M}, \sigma_{M}, \mu_{M}\right)$ by a right-closing map of degree 1 if and only if, in addition, $M$ is aperiodic, $\Delta(M)=\Delta(Q)$ and $c_{M} \Delta(M)=c_{Q} \Delta(Q)$.

The past $\sigma$-algebra $\mathcal{A}_{M}$ of $\left(X_{M}, \sigma_{M}, \mu_{M}\right)$ is generated by the cylinder sets

$$
\left[e_{0}, e_{1}, \ldots, e_{l}\right]_{h}=\left\{x=\left(x_{n}\right) \in X_{M}: x_{h}=e_{0}, x_{h+1}=e_{1}, \ldots, x_{h+l}=e_{l}\right\}
$$

with $l \in \mathbb{Z}^{+}, h \in \mathbb{Z}$ and $h+l \leq 0$. Markov chains $\left(X_{M}, \sigma_{M}, \mu_{M}\right)$ and $\left(X_{Q}, \sigma_{Q}, \mu_{Q}\right)$ are said to be regularly isomorphic if there exists a measure-theoretic isomorphism $\Phi: X_{M} \rightarrow X_{Q}$ such that $\Phi^{-1}\left(\mathcal{A}_{Q}\right) \subset \sigma_{M}^{-N}\left(\mathcal{A}_{M}\right)$ and $\Phi\left(\mathcal{A}_{M}\right) \subset \sigma_{Q}^{-N}\left(\mathcal{A}_{Q}\right)$ for some nonnegative integer $N$.

Theorem 4.3. Let $\left(X_{M}, \sigma_{M}, \mu_{M}\right)$ be a Markov chain and $\left(X_{Q}, \sigma_{Q}, \mu_{Q}\right)$ a Bernoulli shift. The following are equivalent.

(i) $\left(X_{M}, \sigma_{M}, \mu_{M}\right)$ and $\left(X_{Q}, \sigma_{Q}, \mu_{Q}\right)$ are regularly isomorphic.

(ii) There exists a Markov chain $\left(X_{R}, \sigma_{R}, \mu_{R}\right)$ and right-closing factor maps $\phi$ : $\left(X_{R}, \sigma_{R}, \mu_{R}\right) \rightarrow\left(X_{M}, \sigma_{M}, \mu_{M}\right)$ and $\psi:\left(X_{R}, \sigma_{R}, \mu_{R}\right) \rightarrow\left(X_{Q}, \sigma_{Q}, \mu_{Q}\right)$ of degree 1 .

(iii) $M$ is aperiodic, $\beta_{M}=\beta_{Q}, \Delta(M)=\Delta(Q), c_{M} \Delta(M)=c_{Q} \Delta(Q)$ and, considering the $R_{k}^{+}$-matrix $A$ and polynomial $p \in R_{k}^{+}$associated with $M, Q$, there exists a right eigenvector $r$ of $A$ satisfying (a)-(c).

The equivalence of (i) and (ii) of (4.3) is a special case of the main result of $[\mathrm{BT}]$. The equivalence of (ii) and (iii) is deduced from (4.2) with the aid of discrete geometry and techniques adapted from symbolic dynamics.

\section{Combinatorial Formulation}

We have explained that (4.1) and (4.2) may be regarded as statements on rightclosing maps of $\left(X_{A}, \sigma_{A}^{n}\right)$ onto $\left(X_{p}, \sigma_{p}^{n}\right)$. Taking our cues from [BMT, PT], we formulate such maps in terms of state-splittings. (See [LM] for more on statesplittings, which were first employed by Williams.)

Consider an $R_{k}^{+}$-matrix $B$. A state-splitting of $\left(X_{B}, \sigma_{B}\right)$ results from a collection of partitions: For every state $I$ of $G(B)$, the set of edges starting at $I$ is partitioned into sets $(I, 1),(I, 2), \ldots,(I, \rho(I))$. For each $(I, i)$ form a vector $\alpha^{(I, i)}$ indexed by the states of $G(B)$ by letting the $J$ th entry $\alpha^{(I, i)}(J)$ be given by the sum of the weights $\mathrm{wt}_{B}(e)$ of the edges $e$ that belong to $(I, i)$ and terminate at $J$. The sum $\sum_{i=1}^{\rho(I)} \alpha^{(I, i)}$ then equals the $I$ th row of $B$. State-splittings of $\left(X_{B}, \sigma_{B}\right)$ correspond in this way to partitions of rows of $B$ into vectors over $R_{k}^{+}$, leading us to also speak of (state-)splitting $B$. Putting $\widehat{B}((I, i),(J, j))=\alpha^{(I, i)}(J)$, the partition elements index a matrix $\widehat{B}$ such that $\left(X_{\widehat{B}}, \sigma_{\widehat{B}}\right)$ is block isomorphic to $\left(X_{B}, \sigma_{B}\right)$.

Fix $p \in R_{k}^{+}$and an irreducible $R_{k}^{+}$-matrix $A$ with $\beta_{A}=p$. When $A r=p r$ and the vector $r$ is over $R_{k}^{+}$, we will seek state-splittings of a particular form: Writing each entry $r(I)$ as a sum of monomials

$$
r(I)=\sum_{k=1}^{\rho(I)} x^{w(I, i)},
$$

we will seek to partition the $I$ th row of $A^{n}$ into $\rho(I)$ vectors $\alpha^{(I, i)}, 1 \leq i \leq \rho(I)$, such that $\alpha^{(I, i)} \cdot r=x^{w(I, i)} p^{n}$. When we have been able to do this, we say that we 
have a splitting of $A^{n}$ according to $r$. This notion derives its significance from the following.

Theorem 5.1. Let $A$ be an irreducible $R_{k}^{+}$-matrix with $\beta_{A}=p \in R_{k}^{+}$. The following are equivalent.

(i) $\left(X_{p^{n}}, \sigma_{p^{n}}\right)$ is a right-closing factor of $\left(X_{A^{n}}, \sigma_{A^{n}}\right)$ for some $n \in \mathbb{N}$.

(ii) A has a right eigenvector $r$ over $R_{k}^{+}$such that, for some $N \in \mathbb{N}$, the matrix $A^{N}$ can be split according to $r$.

(iii) A has a right eigenvector $r$ over $R_{k}^{+}$such that $A^{n}$ can be split according to $r$ for all large $n$.

(iv) $\left(X_{p}, \sigma_{p}\right)$ is a right-closing eventual factor of $\left(X_{A}, \sigma_{A}\right)$.

With both (4.1) and (4.2), the necessity of the conditions is quickly established with the aid of (5.1). Theorem (5.1) also enables us to use [A] to deduce from (4.1) the sufficiency of the conditions of (4.2): By (4.1) and (5.1), the conditions of (4.2) provide splittings of $A^{n}$ according to $r$ for all large $n$. In [A] Ashley shows how to use analogous splittings and the related factor periodic point condition to produce right-closing maps (of the first powers) in the case of shifts of finite type. A right-closing map of $\left(X_{A}, \sigma_{A}\right)$ onto $\left(X_{p}, \sigma_{p}\right)$ is constructed by going through the argument of $[\mathrm{A}]$ with our factor periodic point condition and splittings of $A^{n}$.

Thus, (4.1)-(4.3) reduce to the construction of a state-splitting of $A^{n}$ according to $r$. This construction takes up much of [MT5] (in the preprint version, 43 Latex pages out of 72). We provide an outline of it.

The right eigenvector $r$ is said to totally conform to $p$ if, in addition to satisfying (a) $-(\mathrm{c})$, it has the property that every entry $r(I) F$-conforms to $p$ for every proper face $F$ of $W(p)$.

The construction actually employs two state splittings. The first uses (b) and (2.1) to prove:

Proposition 5.2. For $n$ large enough, $A^{n}$ may be state-split in such a way that the resulting matrix $\widehat{A^{n}}$ has a right eigenvector $\widehat{r}$ which totally conforms to $p$.

This is followed by the main splitting, outlined in the next section and showing that if a matrix has a totally conforming right eigenvector, then large powers of the matrix can be split according to that eigenvector.

\section{The MAin STATE-SPLitTing}

By (5.1) and (5.2), we may now replace $A$ by a suitable power to assume that $r$ totally conforms to $p$ and that every $F$-component of $A$ is aperiodic for every $F \in \mathcal{F}(p)$. Using (a)-(c) and total conformity, pick $L \in \mathbb{Z}^{+}, u_{F, J} \in \mathbb{Z}^{k}$ and, for each principal component $\mathcal{C}$ and $\operatorname{coset} x^{u} \Delta\left(A_{\mathcal{C}}\right) \in \Delta\left(p_{F}\right) / \Delta\left(A_{\mathcal{C}}\right)$, pick $c_{\mathcal{C}} \in \Delta\left(p_{F}\right)$, $D_{F, J} \in \log \left(\Delta\left(p_{F}\right)\right)$ and polynomials $q^{(F, u)}(J) \in R\left(\Delta\left(A_{\mathcal{C}}\right)\right)$ such that:

(1) $\log (r(J))+u_{F, J} \subset \log \left(p^{L}\right)$ and $\log \left(r(J)_{F}\right)+u_{F, J} \subset \log \left(p_{F}^{L}\right)$ for every $J \in S$

(2) for each principal $F$-component $\mathcal{C}$, the matrix $\widetilde{A}_{\mathcal{C}}$ indexed by (the states of) $\mathcal{C}$ and with

$$
\widetilde{A}_{\mathcal{C}}\left(J, J^{\prime}\right)=A_{\mathcal{C}}\left(J, J^{\prime}\right) \frac{x^{u_{F, J}} x^{D_{F, J}}}{c_{\mathcal{C}} c_{p_{F}} x^{u_{F, J^{\prime}}} x^{D_{F, J^{\prime}}}}
$$

is over $R\left(\Delta\left(A_{\mathcal{C}}\right)\right)$; 
(3) $\sum_{J \in \mathcal{C}} q^{(F, u)}(J) x^{u_{F, J}} x^{D_{F, J}} r(J)_{F}=x^{u} p_{F}^{L}$ for every $F \in \mathcal{F}(p)$, principal $F$ component $\mathcal{C}$ and $x^{u} \Delta\left(A_{\mathcal{C}}\right) \in \Delta\left(p_{F}\right) / \Delta\left(A_{\mathcal{C}}\right)$.

Denote the starting and terminal states of a $G(A)$-path $\gamma$ by $s(\gamma)$ and $t(\gamma)$. Let $W_{n}(I)$ be the set of $G(A)$-paths $\gamma$ of length $n$ and with $s(\gamma)=I$. Let $W_{n}(I, J)$ be the set of $\gamma \in W_{n}(I)$ with $t(\gamma)=J$; and $W_{n}(I, J, w)$ the set of $\gamma \in W_{n}(I, J)$ with $\log (\operatorname{wt}(\gamma))=w$. For $a \in \log \left(r(I) p^{n}\right)$, let $\pi(n, a)$ denote the probability vector of length $\rho(I)$ whose $i$ th entry equals $\pi(n, a, i)=\left(x^{w(I, i)} p^{n}\right)_{a} /\left(r(I) p^{n}\right)_{a}$. The following lemmas make use of (2.3) and (2.4).

Lemma 6.1. Let $\theta, \bar{\epsilon}_{F}>0$ and $D, l \in \mathbb{Z}^{+}$. There exist $\epsilon_{F}>0$ and $N \in \mathbb{N}$ such that

$$
\left|\pi\left(n+l, a+l c_{F}+b, i\right) / \pi(n, a, i)-1\right|<\theta
$$

whenever $n \geq N, \frac{a}{n} \in B\left(F, \epsilon_{F}\right) \backslash B\left(\partial F, \bar{\epsilon}_{F}\right),\left(p^{n}\right)_{a} \neq 0, b \in \log \left(\Delta\left(p_{F}\right)\right)$ and $\|b\| \leq$ $D$.

Lemma 6.2. Let $F \in \mathcal{F}(p), \bar{\epsilon}_{F}>0$ and $\bar{D} \in \mathbb{N}$. There exist $\epsilon_{F}>0$ and $N, \bar{K} \in \mathbb{N}$ such that, for every $n \geq N$ and $a \in \mathbb{Z}^{k}$ with $\frac{a}{n} \in B\left(F, \epsilon_{F}\right) \backslash B\left(\partial F, \bar{\epsilon}_{F}\right)$, we can find a principal F-component $\mathcal{C}, J_{1} \in \mathcal{C}$ and $x^{u} \Delta\left(A_{\mathcal{C}}\right) \in \Delta\left(p_{F}\right) / \Delta\left(A_{\mathcal{C}}\right)$ with

$$
\left|W_{n}\left(I, J_{1}, a-u+b-L c_{F}+u_{F, J_{1}}+D_{F, J_{1}}\right)\right| \geq \frac{1}{\bar{K}}\left(r(I) p^{n}\right)_{a}
$$

whenever $b \in \log \left(\Delta\left(A_{\mathcal{C}}\right)\right)$ and $\|b\| \leq \bar{D}$.

Define the mass of $\gamma \in W_{n}(I)$ to be $\operatorname{mass}(\gamma)=\operatorname{wt}(\gamma) r(t(\gamma))$. For $W \subset W_{n}(I)$, let $\operatorname{mass}(W)$ equal the sum of $\operatorname{mass}(\gamma)$ over $\gamma \in W$. Our goal is to partition $W_{n}(I)$ into sets $(I, i), 1 \leq i \leq \rho(I)$, so that mass $((I, i))=x^{w(I, i)} p^{n}$. We will speak of allocating the elements of $W_{n}(I, J, w)$ according to $\pi(n, w+a)$, where $a \in \log (r(J))$ : What we mean by this is that the number of elements placed in the $i$ th set differs from (the possibly nonintegral) number $\pi(n, w+a, i)\left|W_{n}(I, J, w)\right|$ by less than 1 . Condition (1) guarantees that, any time we assign paths from $W_{n}(I, J, w)$ to the $i$ th set, we have $w+\log (r(J)) \subset \log \left(r(I) p^{n}\right)$.

For the sake of simplicity, we restrict our attention to the case $k=2$.

For each $F \in \mathcal{F}(p)$ and $J$, fix $a_{F, J} \in \log \left(r(J)_{F}\right)$. Write $a_{J}=a_{W(p), J}$. For $F \in \mathcal{F}(p)$, also fix $v_{F} \in \mathbb{Z}^{k}$ with coprime entries which exposes $F$ by having $F \cdot v_{F}=\min \left\{W(p) \cdot v_{F}\right\}$. Write $\delta_{F}=\min \left\{W(p) \cdot v_{F}\right\}=\min \left\{\log (p) \cdot v_{F}\right\}$ and $\delta_{F}(J)=\min \left\{r(J) \cdot v_{F}\right\}$. For $F \in \mathcal{F}(p)$ and $\lambda \in \mathbb{Z}$, let

$$
\mathcal{N}_{n}(F, \lambda)=\left\{a \in \log \left(r(I) p^{n}\right): a \cdot v_{F} \leq \delta_{F}(I)+n \delta_{F}+\lambda\right\} .
$$

Recalling $k=2$, define $\mathcal{N}_{n}\left(\lambda_{0}\right)$ to be the union of $\mathcal{N}_{n}\left(F_{0}, \lambda_{0}\right)$ over all vertices $F_{0}$ of $W(p)$ and define $\mathcal{N}_{n}\left(\lambda_{0}, \lambda_{1}\right)=\mathcal{N}_{n}\left(\lambda_{0}\right) \cup \bigcup_{F_{1}} \mathcal{N}_{n}\left(F_{1}, \lambda_{1}\right)$, where the last union is over all edges $F_{1}$ of $W(p)$. Let $W_{n}(F, \lambda), W_{n}\left(\lambda_{0}\right), W_{n}\left(\lambda_{0}, \lambda_{1}\right)$ respectively denote the union of the sets $W_{n}(I, J, w)$ over all $(J, w)$ such that $w+\log (r(J))$ intersects $\mathcal{N}_{n}(F, \lambda), \mathcal{N}_{n}\left(\lambda_{0}\right), \mathcal{N}_{n}\left(\lambda_{0}, \lambda_{1}\right)$. We drop the subscript from our notation when the length is clear from the context.

Consider $\xi, D \geq 0$ and a partition of $W_{n}\left(\lambda_{0}, \lambda_{1}\right)$ into sets $P(i), 1 \leq i \leq \rho(I)$, satisfying two conditions:

(i) For every $a \in \mathcal{N}_{n}\left(\lambda_{0}, \lambda_{1}\right)$ we have $\operatorname{mass}(P(i))_{a}=\left(x^{w(I, i)} p^{n}\right)_{a}$.

(ii) If $W_{n}(I, J, w) \subset W_{n}\left(\lambda_{0}, \lambda_{1}\right) \backslash W_{n}\left(\lambda_{0}-D, \lambda_{1}-D\right)$, then

$$
1-\xi \leq \frac{\left|W_{n}(I, J, w) \cap P(i)\right|}{\pi\left(n, w+a_{J}, i\right)\left|W_{n}(I, J, w)\right|} \leq 1+\xi
$$


For a suitable choice of the constant $D$, we have:

Lemma 6.3. For every small $\epsilon>0$ there exist $\xi \geq 0$ and $N \in \mathbb{N}$ such that, for any $n \geq N$ and $\lambda_{0}, \lambda_{1}>n \epsilon$, the existence of a partition of $W_{n}\left(\lambda_{0}, \lambda_{1}\right)$ into $\rho(I)$ sets $P(i)$ satisfying (i) and (ii) implies the existence of a partition of $W_{n+L}(I)$ into $\rho(I)$ sets $(I, i)$ such that $\operatorname{mass}((I, i))=x^{w(I, i)} p^{n+L}$.

Sketch of proof. If $W_{n}(I, J, w)$ is not contained in $W_{n}\left(\lambda_{0}, \lambda_{1}\right)$, allocate it according to $\pi\left(n, w+a_{J}\right)$. Let $P^{\prime}(i)$ be the set containing these allocations and $P(i)$. Let $\widetilde{P}(i) \subset W_{n+L}(I)$ be obtained by extending the paths in $P^{\prime}(i)$ :

$$
\widetilde{P}(i)=\left\{\gamma \eta: \gamma \in P^{\prime}(i), \eta \in W_{L}(t(\gamma))\right\}
$$

Then $\operatorname{mass}(\widetilde{P}(i))=\operatorname{mass}\left(P^{\prime}(i)\right) p^{L}$, and $\operatorname{mass}\left(P^{\prime}(i)\right)_{a}=\left(x^{w(I, i)} p^{n}\right)_{a}$ for all $a \in$ $W_{n}\left(\lambda_{0}, \lambda_{1}\right)$. Consider $a \in \log \left(p^{n} r(I)\right) \backslash W_{n}\left(\lambda_{0}, \lambda_{1}\right)$. Taking $F=W(p), x^{u} \Delta(A)=$ $x^{a} \Delta(A)$ in $(3)$, writing $q^{(u)}(J)=q^{(W(p), u)}(J)$ and multiplying by $x^{a-u}$,

$$
\sum_{J} x^{a-u+u_{W(p), J}+D_{W(p), J}} q^{(u)}(J) r(J)=x^{a} p^{L} .
$$

Consider moving, for each $b \in \log \left(q^{(u)}(J)\right)$, exactly $q^{(u)}(J)_{b}$ elements of $W_{n+L}(I, J$, $\left.m y a-u+u_{W(p), J}+D_{W(p), J}+b\right)$ from $\widetilde{P}(j)$ to $\widetilde{P}(i)$. (If $q^{(u)}(J)_{b}<0$, we move the paths from $\widetilde{P}(i)$ to $\widetilde{P}(j)$.) By the above equation, this adds $x^{a} p^{L}$ to $\operatorname{mass}(\widetilde{P}(i))$ and subtracts $x^{a} p^{L}$ from mass $(\widetilde{P}(j))$. To complete the proof, one uses (ii), (6.1) and $(6.2)$ to verify that, for small $\xi$ and large $n$, the sets $\widetilde{P}(j)$ contain the paths required to repeat this "correction" $\left(x^{w(I, i)} p^{n}\right)_{a}-\operatorname{mass}\left(P^{\prime}(i)\right)_{a}$ times.

A partition to which (6.3) applies is constructed by induction. For ease of exposition, we assume $L=0$. This enables us to avoid having, as we did in the sketch of the proof of (6.3), to extend paths by $L$ each time we use (3) to make corrections. For small $\epsilon_{0}>\epsilon_{1}>0$, the induction will have $\epsilon_{0} n+\epsilon_{1} n$ stages. (The choices of $\epsilon_{0}, \epsilon_{1}$ are indicated below.) For $\lambda \in\left\{1, \ldots, \epsilon_{0} n+\epsilon_{1} n\right\}$, we will describe the sets $P(\lambda, i), 1 \leq i \leq \rho(I)$, we have at the end of stage $\lambda$.

For $\lambda \in\left\{1, \ldots, \epsilon_{0} n\right\}$, we only make allocations near vertices of $W\left(p^{n}\right)$ : We start the $\lambda$ th stage having partitioned $W(\lambda-2)=W_{n}(\lambda-2)$ into sets $P(\lambda-1, i)$ with $\operatorname{mass}(P(\lambda-1, i))_{a}=\left(x^{w(I, i)} p^{n}\right)_{a}$ for all $a \in \mathcal{N}(\lambda-2)$. Then, for each vertex $F_{0}$, allocate according to $\pi\left(n, w+a_{F_{0}, J}\right)$ each $W(I, J, w)$ contained in $W\left(F_{0}, \lambda-\right.$ $1) \backslash W(\lambda-2)$. According to (2.2), by picking $\epsilon_{0}$ small and $n$ large, we can be sure that for $a \in \mathcal{N}\left(F_{0}, \lambda-1\right) \backslash \mathcal{N}(\lambda-2)$ the coefficient $\operatorname{mass}(P(\lambda-1, i))_{a}$ will be an arbitrarily small fraction of $\left(x^{w(I, i)} p^{n}\right)_{a}$. Combining this with (6.2), we are assured of the supply of paths needed to use (3) to make corrections and obtain $P(\lambda, i)$ with $\operatorname{mass}(P(\lambda, i))_{a}=\left(x^{w(I, i)} p^{n}\right)_{a}$ for all $a \in \mathcal{N}(\lambda-1)$.

From $\lambda=\epsilon_{0} n+1$, we allocate paths near the edges of $W\left(p^{n}\right)$ as well. For $\lambda \in\left\{\epsilon_{0} n+1, \ldots, \epsilon_{0} n+\epsilon_{1} n\right\}$ the $\lambda$ th stage consists of $d$ steps. (The choice of $d$ will become clear in a minute. $)$ We start the $\lambda$ th stage having partitioned $W(\lambda-$ $\left.2, \lambda-\epsilon_{0} n-2\right)$ into sets $P(\lambda-1, i)$ with $\operatorname{mass}(P(\lambda-1, i))_{a}=\left(x^{w(I, i)} p^{n}\right)_{a}$ for all $a \in \mathcal{N}\left(\lambda-2, \lambda-\epsilon_{0} n-2\right)$. In step 1, we allocate and correct near edges: For each edge $F_{1}$, allocate according to $\pi\left(n, w+a_{F_{1}, J}\right)$ each $W(I, J, w)$ contained in $W\left(F_{1}, \lambda-\epsilon_{0} n-1\right) \backslash W\left(\lambda-2, \lambda-\epsilon_{0} n-2\right)$. Then use (3) to correct: We employ (2.2) and (6.2) as before, this time applying them to edges. In addition, we use (6.1) to see that errors due to the choice of $a_{F_{1}, J}$ may be corrected. While getting the coefficients correct for $a \in \mathcal{N}\left(F_{1}, \lambda-\epsilon_{0} n-1\right)$, step 1 may perturb those of boundedly 
many $a \in \mathcal{N}(\lambda-2) \backslash \mathcal{N}(\lambda-d)$. Steps 2 to $d-1$ make up for the perturbations. (Step $\mu \in\{2, \ldots, d-1\}$ gets $\mathcal{N}(\lambda-d+\mu-1)$ correct.) Applying (2.2) to edges, for small $\epsilon_{1}$ and large $n$, the perturbations will be small enough for us to correct them by employing (3) near vertices. Finally, step $d$ allocates and corrects at $a \in \mathcal{N}(\lambda-1) \backslash \mathcal{N}\left(\lambda-2, \lambda-\epsilon_{0} n-1\right)$ to yield $P(\lambda, i)$ with $\operatorname{mass}(P(\lambda, i))_{a}=\left(x^{w(I, i)} p^{n}\right)_{a}$ for all $a \in \mathcal{N}\left(\lambda-1, \lambda-\epsilon_{0} n-1\right)$.

\section{REFERENCES}

[A] J. Ashley, Resolving factor maps for shifts of finite type with equal entropy, Ergod. Th. and Dynam. Sys. 11 (1991), 219-240. MR 92d:58056

[AMT] J. Ashley, B. Marcus and S. Tuncel, The classification of one-sided Markov chains, Ergod. Th. and Dynam. Sys. 17 (1997), 269-295. MR 98k:28021

[BMT] M. Boyle, B. Marcus and P. Trow, Resolving maps and the dimension group for shifts of finite type, Mem. Amer. Math. Soc. 377 (1987). MR 89c:28019

[BT] M. Boyle and S. Tuncel, Regular isomorphism of Markov chains is almost topological, Ergod. Th. and Dynam. Sys. 10 (1990), 89-100. MR 92i:28021

[H] D. Handelman, Positive polynomials and product type actions of compact groups, Mem. Amer. Math. Soc. 320 (1985). MR 86h:46091

[LM] D. Lind and B. Marcus, An Introduction to Symbolic Dynamics and Coding, Cambridge Univ. Press, Cambridge, 1995. MR 97a:58050

[M] B. Marcus, Factors and extensions of full shifts, Monatshefte Math. 88 (1979), 239-247. MR 81g:28023

[MT1] B. Marcus and S. Tuncel, The weight-per-symbol polytope and scaffolds of invariants associated with Markov chains, Ergod. Th. and Dynam. Sys. 11 (1991), 129-180. MR 92g:28038

[MT2] B. Marcus and S. Tuncel, Entropy at a weight-per-symbol and embeddings of Markov chains, Invent. Math. 102 (1990), 235-266. MR 91k:28023

[MT3] B. Marcus and S. Tuncel, Matrices of polynomials, positivity, and finite equivalence of Markov chains, J. Amer. Math. Soc. 6 (1993), 131-147. MR 93e:28022

[MT4] B. Marcus and S. Tuncel, On large powers of positive polynomials in several variables, preprint.

[MT5] B. Marcus and S. Tuncel, Resolving Markov chains onto Bernoulli shifts, preprint.

[O] D. Ornstein, Ergodic Theory, Randomness and Dynamical Systems, Yale Univ. Press, New Haven, 1974. MR 56:5836

[PS] W. Parry and K. Schmidt, Natural coefficients and invariants for Markov shifts, Invent. Math. 76 (1984), 15-32. MR 86b:28022a

[PT] W. Parry and S. Tuncel, On the stochastic and topological structure of Markov chains, Bull. London Math. Soc. 14 (1982), 16-27. MR 84i:28024

[T] S. Tuncel, Faces of Markov chains and matrices of polynomials, Contemp. Math., Vol. 135, Amer. Math. Soc., Providence, 1992, pp. 391-422. MR 94m:28034

IBM Almaden Research Center, 650 Harry Road, San Jose, CA 95120

E-mail address: marcus@almaden.ibm.com

Department of Mathematics, Box 354350, University of Washington, Seattle, WA 98195

E-mail address: tuncel@math.washington.edu 